\title{
Galaxy formation from dry and hydro simulations
}

\author{
Luca Ciotti \\ Department of Astronomy, University of Bologna, via Ranzani 1, 40127 Bologna, Italy \\ email: luca.ciotti@unibo.it
}

\begin{abstract}
The effects of dry and wet merging on the Scaling Laws (SLs) of elliptical galaxies (Es) are discussed. It is found that the SLs, possibly established at high redshift by the fast collapse of gas-rich and clumpy stellar distributions in preexisting dark matter halos following the cosmological SLs, are compatible with a (small) number of galaxy mergers at lower redshift.
\end{abstract}

Keywords. Galaxies: elliptical and lenticular, cD - Galaxies: formation - Galaxies: evolution

The main results obtained in a series of papers (Ciotti \& van Albada 2001; Nipoti, Londrillo \& Ciotti 2003; Lanzoni et al. 2004; Ciotti, Lanzoni \& Volonteri 2007; see also Ciotti 2009), are presented. It is found that 1) Parabolic dry merging in a population of low mass spheroids leads to massive Es that fail the Faber-Jackson (FJ) and Kormendy relations, being characterized by low velocity dispersion and very large effective radii. Parabolic wet merging in the same population of progenitors leads to Es in better agreement with the observed SLs, as long as enough gas for dissipation is available. 2) The edge-on structure of the Fundamental Plane (FP) is surprisingly preserved. Therefore, the FJ and Kormendy relations, despite their larger scatter, are stronger tests for merging than the edge-on FP. 3) Parabolic dry or wet merging of Es following the observed SLs over the full mass range preserve the Kormendy, FJ, and edge-on FP relations. Thus, massive Es cannot be formed by parabolic merging of low mass spheroidal galaxies, even in presence of substantial gas dissipation, but their SLs, once established by galaxy formation, are robust against merging. 4) Dark matter halos obtained from cosmological simulations define a FJ, a Kormendy, and a FP-like relation, as expected from the spherical collapse model for virialized systems. 5) Numerical simulations of cold dissipationless collapse in pre-existing dark matter halos can reproduce Sersic profiles remarkably similar to those observed, over a large radial range. Note that cold dissipationless collapse is a process which is expected to dominate the late stages of an initially dissipative process. Thus the SLs of Es, possibly established at high redshift by the fast collapse of gas rich and clumpy stellar distributions in pre-existing dark matter halos (following the cosmological SLs), can persist even in the presence of a moderate number of dry or wet mergings. Then monolithic-like collapse at early times and subsequent merging could just represent the different phases of galaxy formation (collapse) and evolution (merging, in addition to the aging of the stellar population and related phenomena).

\section{References}

Ciotti, L. 2009, La Rivista del Nuovo Cimento 32, n.1, 1

Ciotti, L. \& van Albada, T. S. 2001, ApJ 552, L13

Ciotti, L., Lanzoni, B., \& Volonteri, M. 2007, ApJ 658, 65

Lanzoni, B., Ciotti, L., Cappi, A., \& Tormen, G. 2004, ApJ 600, 640

Nipoti, C., Londrillo, P., \& Ciotti, L. 2003, MNRAS 342, 501 